\title{
Mass transport improvement in microscale using diluted ferrofluid and a non-uniform magnetic field
}

\author{
Majid Hejazian, ${ }^{a}$ Dinh-Tuan Phan, ${ }^{b}$ Nam-Trung Nguyen ${ }^{a, *}$
}

Received 00th April 2016

Accepted 00th xxxx 2016

DOI: $10.1039 / \times 0 \times x 00000 x$

www.rsc.orgl

\begin{abstract}
This paper demonstrates that a non-uniform magnetic field and diluted ferrofluid can improve mass transport of diamagnetic solutes in a microfluidic device. A hydrodynamic flow-focusing configuration was employed to test this hypothesis. The system consists of a core stream and two sheath streams. The core stream contains a diluted ferrofluid and a fluorescent dye. Without a magnetic field, both magnetic nanoparticles of the ferrofluid and the fluorescent dye in the core stream rely on molecular diffusion to spread transversally. The non-uniform magnetic field of a permanent magnet causes magnetophoresis of the magnetic nanoparticles, magnetoconvective secondary flow and subsequently the mass transport of the diamagnetic fluorescent dye. We observed a significant enhancement in mass transport of the fluorescent dye. The platform presented here could be used as a microfluidics-based gradient generator or micromixer.
\end{abstract}

\section{Introduction}

Over the last two decades, microfluidics has proven to be a technology that provides highly effective tools for biological and chemical research. ${ }^{1,2}$ This technology has been exploited for designing a diverse range of fluid handling devices in the microscale such as micropumps, ${ }^{3}$ microvalves, ${ }^{4}$ micromixers, ${ }^{5}$ and microseparators. ${ }^{6,7}$ Micro gradient generator and micromixer are the two typical microfluidic devices that rely on effective mass transport in the microscale, but are currently limited to molecular diffusion due to the predominant laminar flow in this size scale.

Recently, microfluidics-based concentration gradient generators ${ }^{8}$ have been used for cell migration studies. A concentration gradient generator is capable of creating a gradient of biochemical signals such as growth factors, hormones and chemokines. ${ }^{9}$ Pressure-driven gradient generator is one of the most common types in continuousflow microfluidics, where mass transport is governed by molecular diffusion and convection through a network of microchannels. Lung cancer chemotherapy resistance, for instance, has been studied using this device. ${ }^{10}$ An integrated microfluidic device having an upstream gradient network and downstream cell culture chambers was used for this purpose. Other type of gradient generators relies solely on molecular diffusion. An example of this type is a device for bacterial chemotaxis analysis, the movement of cells in a channel induced by the cells chemotactic response. ${ }^{11}$ The diffusion of a chemical through a porous membrane adjacent to the channel wall formed a static linear chemical gradient in the microchannel.

Micromixers are other microfluidic devices that play an important role for biological and chemical research. Micromixers can be categorised in passive and active groups. ${ }^{5}$ Passive micromixers solely rely on diffusion or chaotic advection for mixing, while active micromixers take advantage of an external energy field, such as temperature, magnetic field, electric field and acoustic field. Parallel lamination micromixers are a type of passive micromixers that has been exploited to study diffusive mixing in microchannels. $\mathrm{Wu}$ et al. developed a analytical model and used a diluted fluorescent dye to evaluate the concentration distribution across a microchannel width. ${ }^{12}$ The authors further examined analytically and experimentally diffusive mixing in hydrodynamic flow-focusing configuration with three fluid streams. ${ }^{13}$ The concentration was measured using recorded images of a fluorescent dye, while the velocity distribution was determined with micro particle image velocimetry (micro-PIV). 


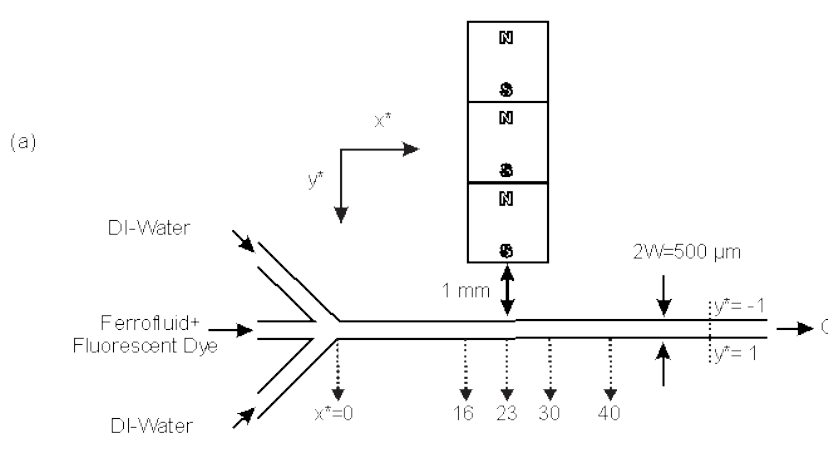

(b)

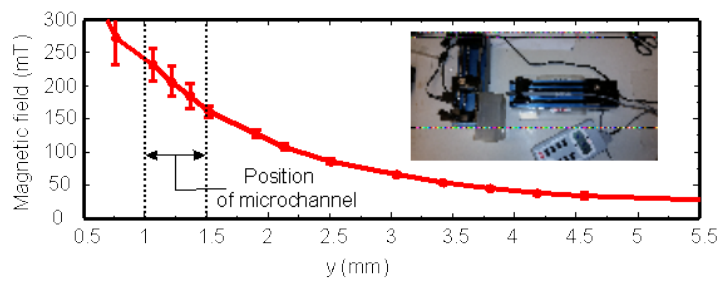

Fig. 1 (a) Schematic of the setup with a microchannel and a set of permanent magnets. (b) Characterisation of magnetic field. The inset shows the setup used for magnetic field measurement. The distance was adjusted using the linear stage of a syringe pump $\left(x^{*}=2 x / W, y^{*}=2 y / W\right)$.

Active mixing employs externally induced disturbance to improve mass transport. For instance, magnetofluidic micromixers utilize a magnetic field and magnetic particles suspended in a fluid to enhance mass transport through magnetophoresis, the migration of magnetic particles. Wang et al. investigated numerically a magnetofluidic micromixer using magnetic beads for fluid agitation. ${ }^{14}$ Tsai et al. and Fu et al. used a permanent magnet and ferrofluid, a liquid with a suspension of magnetic nanoparticles, to improve the performance of a Y-mixer. ${ }^{15,16}$ The non-uniform magnetic field re-distributes the magnetic nanoparticles in the microchannel and consequently induces magnetoconvective flow. Mixing efficiency between water and ferrofluid can be higher than $90 \%$, which is significant compared to the value of below $15 \%$ for pure molecular diffusion in the same microchannel. Azimi et al. utilised ferrofluid and a stationary magnetic field to enhance mixing in an Y- micromixer. ${ }^{17}$ The mixing efficiency can reach up to $70 \%$ with an optimal concentration of magnetic nanoparticles. Our group previously exploited an uniform magnetic field and the susceptibility gradient between a diamagnetic fluid and a ferrofluid to induce mixing in a circular chamber. ${ }^{18,}{ }^{19}$ The gradient of magnetic susceptibility led to instability at the liquid-liquid interface and consequent mixing. In a recent work, we demonstrated that even without the existence of a magnetic susceptibility gradient, mixing could be achieved with a non-uniform magnetic field. ${ }^{20}$ The field of a permanent magnet induces a body force on the ferrofluid inside the chamber, resulting in a secondary flow called magnetoconvection. The magnetoconvective transport was traced by both fluorescent dye and diamagnetic particles.

As discussed above, mixing of ferrofluid and water has been reported, but mass transfer enhancement for other diamagnetic species such as fluorescent dye through magnetic field have not been studied and reported before. In the present work, we employed permanent magnets in a simple hydrodynamic focusing device to create a non-uniform magnetic field. The transport process of the fluorescent dye was evaluated based on the recorded fluorescent images. The concentration field was analysed for different flow rates to compare the relative significance of hydrodynamic transport and the secondary magnetoconvective transport induced by the magnetic field. A numerical study was also carried out to simulate the concentration field and velocity field in the absence of magnetic field. Magnetically induced mass transfer was observed and discussed based on our experimental results. The enhancement in mass transfer is benchmarked by the convective/diffusive flowfocusing system that relies on molecular diffusion only.

\section{Materials and methods}

Fig. 1(a) shows the schematic of the hydrodynamic focusing device. The microchannel has a depth of $H=50 \mu \mathrm{m}$, a width of $W=500 \mu \mathrm{m}$, and a length of $L=12 \mathrm{~mm}$. The device was fabricated in polydimethylsiloxane (PDMS) using the standard soft lithography technique. Detailed information about the fabrication procedure can be summarized as follows. ${ }^{21,22}$ First, photoresist SU-8 with a thickness of $50 \mu \mathrm{m}$ was coated evenly and patterned on a Si wafer by a standard photolithography process. A mixture of Sylgard 184 base and a curing agent (Dow Corning Inc.) at 10:1 ratio by weight was poured onto the SU-8 mould and subsequently degassed for one hour in a vacuum chamber. A 2-hour baking at $80{ }^{\circ} \mathrm{C}$ in the oven followed. Subsequently, the top PDMS layer was peeled off from the SU-8 mould and the 1-mm access holes were punched. The PDMS microchannel was bonded to another flat PDMS base with the help of oxygen plasma treatment and underwent another post-baking process at $80^{\circ} \mathrm{C}$ for 10 minutes to enhance the bonding strength.

Three precision syringe pumps (SPM100, SIMTech Microfluidics Foundry) delivered the fluids into the microfluidic device. The whole setup was placed on an inverted microscope (Nikon Eclipse TS 100) equipped with a high-speed camera (Photron 120K-M2) for visualization. Diluted ferrofluid mixed with fluorescent dye was used for the core stream. For this purpose, 0.05 gr of fluorescein sodium salt (acid yellow, Sigma-Aldrich Co.) was dissolved in $20 \mathrm{ml}$ of DI-water. The water-based ferrofluid (EMG707, Ferrotec) was diluted to $20 \%$ vol. of the commercially available solution using the prepared fluorescein solution. Three identical 3.2- $\mathrm{mm}^{3}$ neodymium-iron-boron (NdFeB) permanent magnets (B222, K\&J Magnetics Inc.) provided the magnetic field for this experiment. DI-water served as the liquid in both sheath streams. The experiments were carried out with a constant flow rate ratio, for three different flow rates (30:2.5:30, 60:5:60, 120:10:120 $\mu \mathrm{L} / \mathrm{min})$. The magnetic field of the permanent magnets was measured and calibrated using a gauss meter (Hirst Magnetic Instruments Ltd). Fig. 1(b) shows the measured magnetic flux density as a function of the distance from the edge of the magnet. The distance between the magnets to channel wall was $1 \mathrm{~mm}$. Across the microchannel width, the magnetic flux density drops from approximately $250 \mathrm{mT}$ to175 mT.

\section{Numerical simulation}

We used diffusive/convective transport as the reference for the magnetoconvective/convective transport that is characterized later experimentally. A numerical model with COMSOL (COMSOL Inc., USA) simulated the diffusive/convective transport in the flowfocusing configuration to predict the distribution of fluorescent dye in the microchannel, Fig. 1(a). The two-dimensional model consists of three inlets, a straight rectangular channel and one outlet. Because of the low aspect ratio between channel height and channel width, 
a)

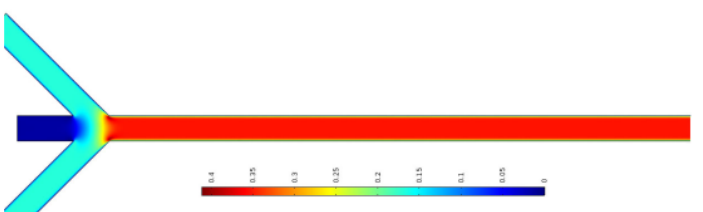

b)

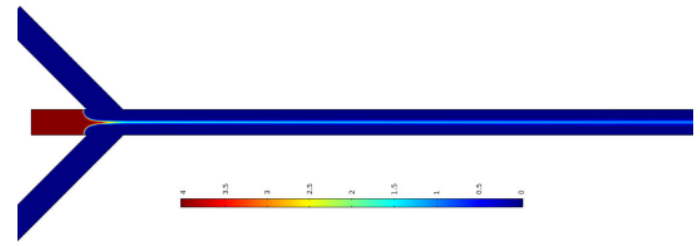

Fig. 2 Simulation results: (a) velocity field; (b) concentration distribution at the lower flow rate of $30: 2.5: 30 \mu \mathrm{L} / \mathrm{min}$, and $D=1 \times 10^{-9} \mathrm{~m}^{2} / \mathrm{s}$.

the velocity is averaged in the z-axis. All streams were considered as incompressible. Steady-state conditions were applied. The model solves the continuity equation:

$\nabla \cdot\left(\rho_{\mathrm{f}} u_{\mathrm{f}}\right)=0$

and the Navier-Stokes equation:

$\rho_{\mathrm{f}}\left(u_{\mathrm{f}} \cdot \nabla\right) u_{\mathrm{f}}=-\nabla P+\nabla \cdot\left(\eta \cdot \nabla u_{\mathrm{f}}\right) \quad$,

where $P(\mathrm{~Pa})$ is the pressure, $u_{\mathrm{f}}(\mathrm{m} / \mathrm{s})$ is the fluid velocity, and $\rho_{\mathrm{f}}$ $\left(\mathrm{kg} / \mathrm{m}^{3}\right)$ and $\eta$ (Pa.s) are the density and dynamic viscosity of the fluid, respectively. The normal inflow velocities are set for the three inlets, no slip conditions were considered at the walls, and pressure has no viscous stress at the outlet. Using single phase model, the density of the ferrofluid is calculated by ${ }^{23}$ :

$\rho_{\mathrm{f}}=(1-\varphi) \rho_{\mathrm{water}}+\varphi \rho_{\mathrm{np}}$

where $\rho_{\text {np }}$ and $\varphi$ are the density of nanoparticles and volume fraction, respectively. The effective dynamic viscosity of the ferrofluid is estimated $\mathrm{as}^{24}$ :

$\mu_{\mathrm{f}}=\mu_{\text {water }}\left(\frac{1}{(1+\varphi)^{0.25}}\right)$

Diffusive/convective transport of a solute is obtained by solving:

$-\nabla \cdot(-D \nabla c+c u)=0$

where $c\left(\mathrm{~mol} / \mathrm{m}^{3}\right)$ is the concentration, and $D\left(\mathrm{~m}^{2} / \mathrm{s}\right)$ is the diffusion coefficient of the solute. The concentrations for the middle inlet and the sheath inlets were set to $4 \mathrm{~mol} / \mathrm{m}^{3}$ and $0 \mathrm{~mol} / \mathrm{m}^{3}$ respectively. For the numerical solution of the above equations, finite element discretization was based on second order functions for velocity, while the pressure and concentration fields are described by linear basis functions. We used the fine mesh consisting of 64324 domain elements and 1976 boundary elements. As mentioned above, the two-dimensional (2D) model considers the depth-averaged velocity. The numerical simulation was carried out for three flow rates at a fixed ratio as used in the later experiments. Figure 2 shows the representative results for flow field and concentration field.

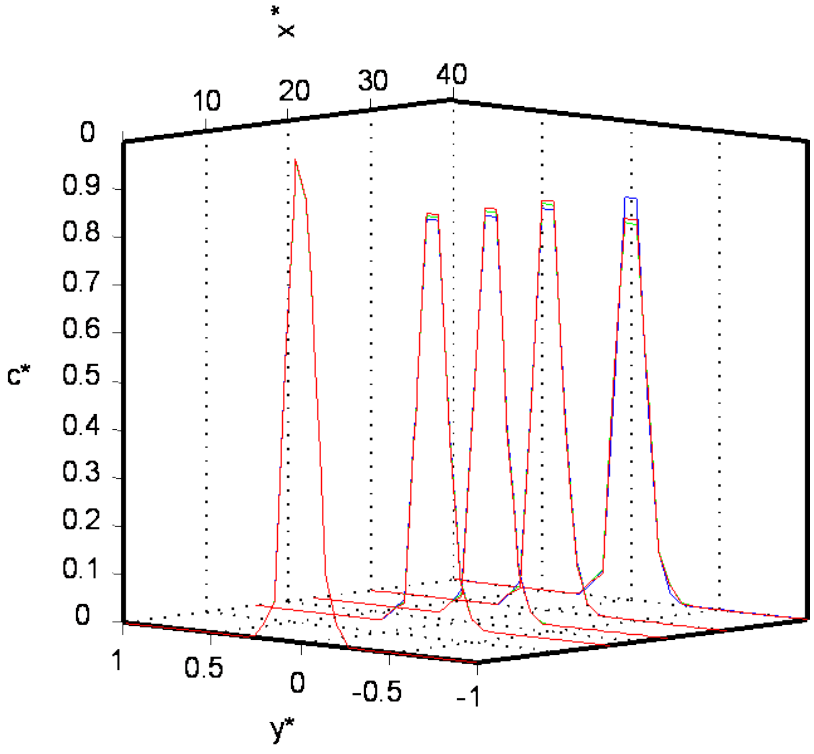

Fig. 3 Simulation results: concentration profiles along the channel for all flow rates: low flow rate (blue), medium flow rate (green), and high flow rate (red).

\section{Results and discussion}

The experiments were carried out for a constant flow rate ratio between the sheath and core streams but different total flow rates, to vary the strength of convective transport. Diluted ferrofluid mixed with fluorescent dye serves as the fluid of the core stream. DI-water works as the sheath fluids sandwiching the core stream in the microchannel. Our aim is to investigate the transport and spreading of the core flow in response to the magnetic field. Without a magnetic field, the width of the core flow (measured as the width between the maximum concentration gradients) is expected to remain constant, as the flow rates and the viscosity determine this width only. We also aim to evaluate the effect of magnets on the mass transport of the fluorescent dye. Six images were taken at different locations along the channel: $x=0,4,5.75,7.5,10,12 \mathrm{~mm}$. The magnets were located between $x=4 \mathrm{~mm}$ to $x=7.5 \mathrm{~mm}$. We normalised the length scale by half of the channel width $W / 2$ $\left(x^{*}=2 x / W, y^{*}=2 y / W\right)$. Half channel width $W / 2$ was used as the characteristic length because we later use the location $y^{*}=0$ as the centre line of the microchannel. The negative space $\left(y^{*}<0\right)$ is next to the magnet and exposed to the field of the permanent magnets.

Fig. 3 shows the simulated concentration distribution at locations along the channel length for different flow rates as used later in the experiments. Without a magnet, the concentration has its maximum

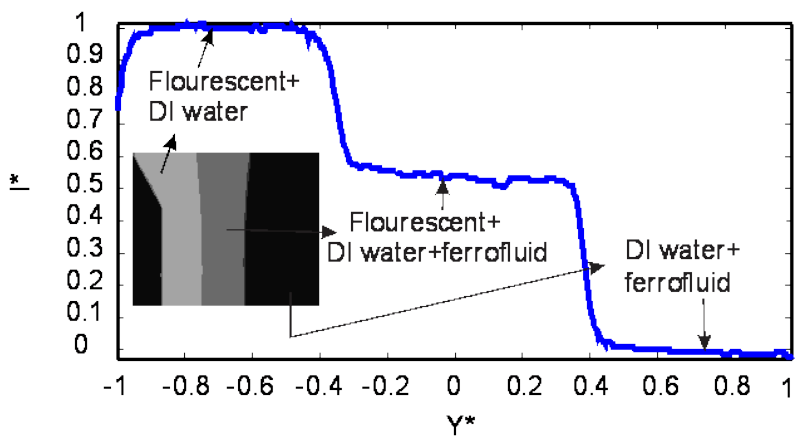

Fig. 4 The calibration results: normalized intensity versus $y^{*}$. The inserted image is the one used for the evaluation of intensity at $x^{*}=0$. 


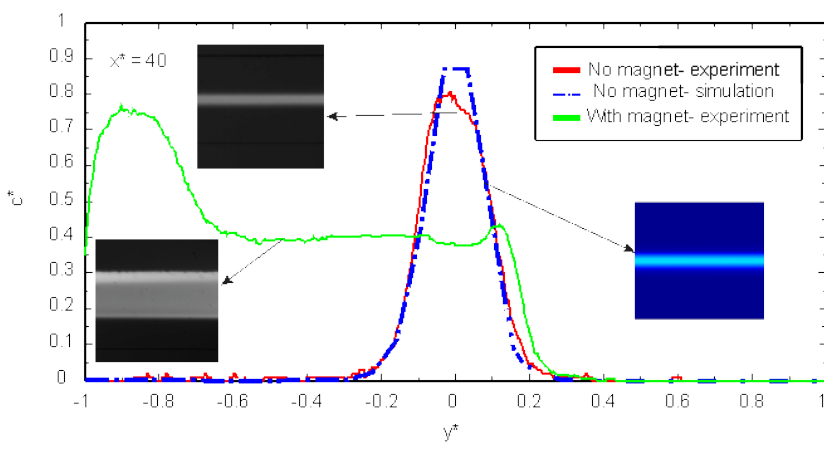

Fig. 5 A comparison between simulation and experimental results for the lower flow rate of $30: 2.5: 30 \mu \mathrm{L} / \mathrm{min}, D=1 \times 10^{-9} \mathrm{~m}^{2} / \mathrm{s}$ and at $x^{*}=40$.

value at the centre line of the channel $\left(y^{*}=0\right)$. The profiles for different flow rates are almost overlapping because the flow rate ratio was kept constant. A diffusion coefficient of $D=1 \times 10^{-9} \mathrm{~m}^{2} / \mathrm{s}$ was used in our simulation. ${ }^{25}$

We also carried out a calibration of fluorescent intensity, to understand the light-blocking role of magnetic nanoparticles on the fluorescent intensity. The intensity calibration corrects the intensity value and converts it into the dimensionless concentration $c^{*}$ of the fluorescent dye. Three streams comprising DI-water mixed with Fluorescein dye, ferrofluid mixed with Fluorescein dye, and ferrofluid are fed into the microchannel with identical flow rates. Fig. 4 depicts the normalized intensity across the channel width. Fig. 4 shows that the ratio between the intensity of ferrofluid/fluorescent dye to DI-water/fluorescent dye mixture is around 0.55 .

Fig. 5 compares the experimental results at $x^{*}=40$ and at the lower flow rate of $2.5 \mu \mathrm{L} / \mathrm{min}$ and $30 \mu \mathrm{L} / \mathrm{min}$ for the core and sheath flows, respectively. In the absence of a magnetic field, experimental and numerical data agree relatively well. In the presence of a magnetic field, substantial transport of fluorescent dye toward the magnets was observed. The mass transport of fluorescent dye was obviously enhanced in the presence of the permanent magnets. The magnetic nanoparticles move towards the magnets as result of the magnetophoretic effect. The redistribution of magnetic nanoparticles leads to susceptibility mismatch between the sheath flow and the core stream. Both magnetic field gradient and magnetic susceptibility gradient lead to a body force that causes the so-called magnetoconvective secondary flow. This secondary flow transports along the dye molecules, which are too small to be affected by diamagnetophoretic effect.

Next, we evaluated the fluorescent intensity across the channel width, to examine the extent of the transport of fluorescent dye. Fig. 6 shows the normalised intensity distribution of all positions along the $x^{*}$ axis for the three different total flow rates mentioned above. The results consistently show that the dye molecules moved toward the permanent magnet, and followed the same path of the magnetic nanoparticles. At low and medium total flow rates, the dye reached and accumulated at the top channel wall $\left(y^{*}=-1\right)$. As the total flow rate increases, the pressure-driven hydrodynamic flow competes with magnetically driven secondary flow, and the intensity gradient becomes steeper. At the higher flow rate, the fluorescent dye could not reach the top channel wall.
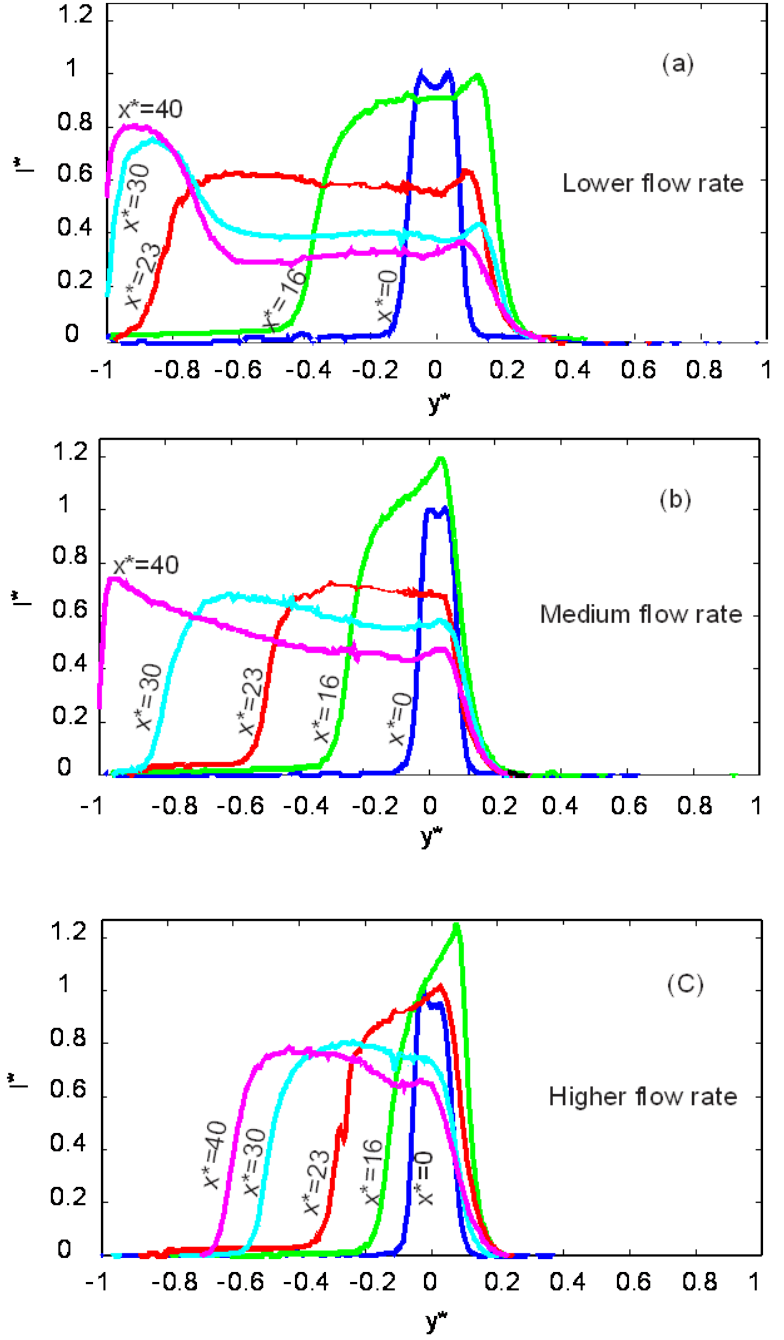

Fig. 6 Normalized intensity versus $y^{*}$ axis for six $x^{*}$ positions along the channel ( $x^{*}=0$ : blue, $x^{*}=16$ : green, $x^{*}=23$ : red, $x^{*}=30$ : cyan, $x^{*}=40$ : purple,) and three flow different flow rates: (a) 30-2.5-30; (b) 60-5-60; (c)120-10120.

Fig. 6 also indicates that the magnetic field has no effect on the concentration distribution of the dye at the inlet $\left(x^{*}=0\right)$. The distribution of the fluorescent dye is similar to that numerically simulated and previously reported. ${ }^{12}$ The mass transport of the dye in the channel width direction relies on molecular diffusion only. The saddle shape on top of the intensity profiles at $x^{*}=0$, is the result of the butterfly effect ${ }^{26}$ and the different diffusion coefficients of fluorescent dye and magnetic nanoparticles. The smaller dye molecules, having a larger diffusion coefficient, migrate more into the sheath streams of DI-water, while magnetic nanoparticles with a diffusion coefficient of $D=4.29 \times 10^{-11} \mathrm{~m}^{2} / \mathrm{s}$, three orders of magnitude smaller then that of fluorescent dye do not diffuse significantly. As a result, the centre of the core stream appears darker than its border.

A further interesting phenomenon could be observed from the intensity profiles in Fig. 6. As the maximum intensity is expected at the channel inlet, where species in the core stream have not diffused into the sheath steams. However, at $x^{*}=16$ (medium flow rate) and 

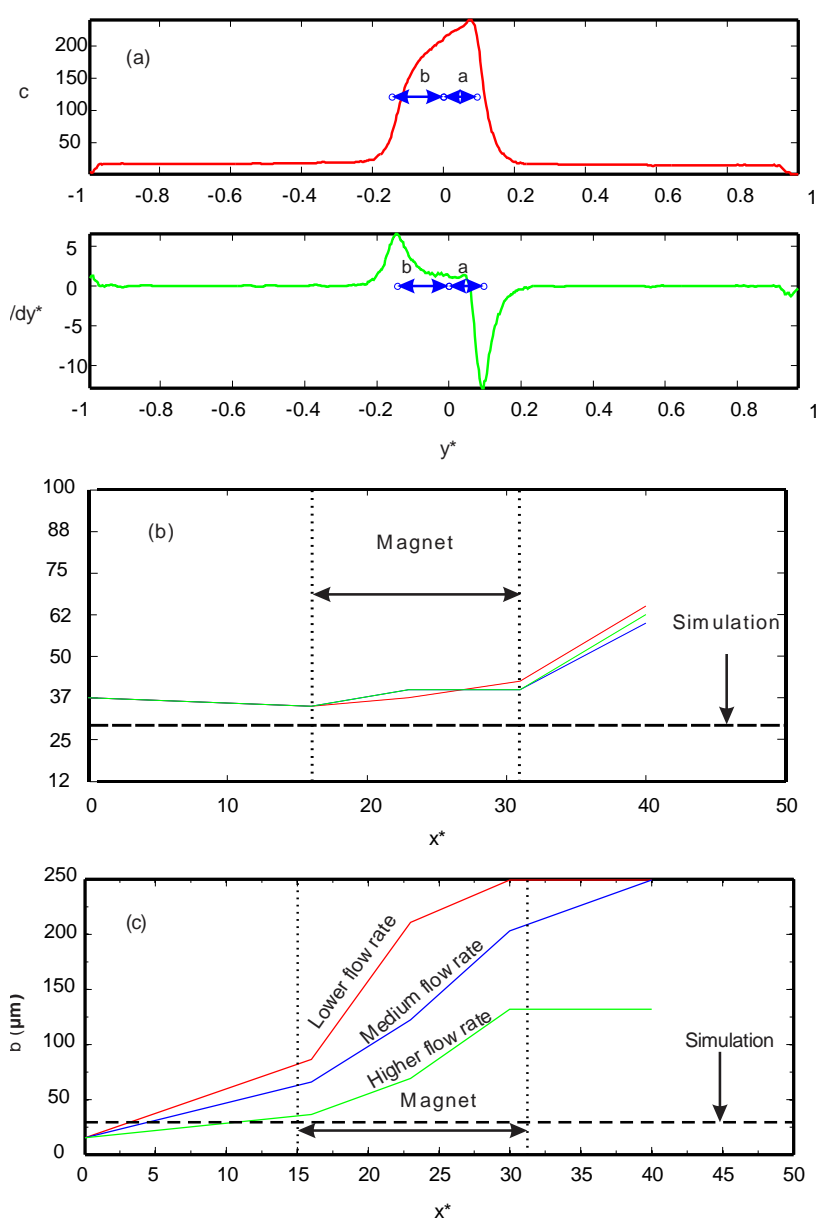

Fig. 7 Evaluation of the width of the core stream: (a) The width is evaluated as the distance between the gradient peak to the centreline $\left(y^{*}=0\right)$; (b) Spreading width at $y^{*}>0$ along the channel; c) Spreading width at $y^{*}<0$ along the channel. Low flow rate: red, medium flow rate: blue, high flow rate: green

$x^{*}=16,23$ (high flow rate), the intensity peak exceeds the maximum intensity of the inlet, and moves away from the magnets. This phenomenon could be explained by the migration of the dark iron oxide nanoparticles toward the magnets due to magnetophoresis. The migration dilutes the ferrofluid at the positions in the proximity of the interface between the core and the sheath streams. Consequently, the remaining fluorescent dye appears to be brighter without the light-blocking magnetic nanoparticles.

At positions near the outlet, two intensity peaks can be observed. The lower one at $y^{*}>0$ represents a more concentrated fluorescein line at the interface. The butterfly effect at the inlet and the absence of blocking magnetic nanoparticles created this bright line. As more magnetic nanoparticles moved toward the permanent magnet in downstream locations $\left(x^{*}=16,23,30\right)$, this peak appears to move to the left and approaches $y^{*}=0$.

The second peak on the left side $\left(y^{*}<0\right)$ is higher compared to the right peaks. This peak appears next to the wall on the side of the magnets. As mentioned above, fluorescent dye is transported toward the magnet by magnetoconvective secondary flow and accumulates on the channel wall. The spread of fluorescent dye on the left side $\left(y^{*}<0\right)$ confirms that magnetoconvective transport induced by the magnetic field is the dominant mass transfer mechanism in this flow configuration. Fluorescent dye does not spread to the right side $\left(y^{*}>0\right)$, due to the insignificant molecular diffusion relative to the pressure-driven convective transport.

We further evaluated the spreading width of the core stream. Fig. 7(a) describes how the spreading width is determined from the maximum intensity gradients at the interface between core and sheath streams. Fig. 7(b) plots the width $a$ at $y^{*}>0$ along the channel length $x^{*}$. Fig. 7(c) shows the width $b$ at $y^{*}<0$ along the channel lengh $x^{*}$. Fig. 7(c) indicates that at the lower flow rate the spreading width for the left part $\left(y^{*}<0\right)$ quickly reaches the value of the half width $W / 2$. As the flow rate increases, the spreading width $b$ decreases, as the pressure-driven hydrodynamic flow dominates over the magnetoconvective flow.

\section{Conclusions}

We used a flow focusing system with three streams to investigate the mass transport of fluorescein dye in a microchannel using diluted ferrofluid and an external nonuniform magnetic field. The sheath streams were diamagnetic DI water, while the core stream was diluted ferrofluid. Fluorescein dye was dissolved in the core stream to trace the mass transport of the secondary magnetoconvective flow. Various phenomena were involved in the transport process of the fluorescent dye: magnetophoresis of magnetic nanoparticles, magnetoconvective secondary flow due to the magnetic field gradient and the gradient of magnetic susceptibility, molecular diffusion, and pressure-driven hydrodynamic flow. The magnetophoresis effect on magnetic nanoparticles redistributes their concentration leading to a gradient in magnetic susceptibility. This susceptibility gradient of the fluid together with the magnetic field of the permanent magnet causes a bulk force acing on the fluid and consequently the magnetoconvective flow. As shown in our experiments, the transport process of fluorescent dye caused by magnetoconvective secondary flow is comparable to that of the pressure-driven hydrodynamic flow and significantly higher than molecular diffusion. The significance of the observed phenomenon is that transport of a diamagnetic sample transversal to the main pressure-driven flow could be improved by orders of magnitude using permanent magnets and diluted ferrofluid. The observed phenomenon has potential applications in designing more efficient micro-mixers or gradient generators.

\section{Acknowledgements}

The authors acknowledge the Australian Research Council Linkage grant LP150100153 to NTN and the international PhD scholarship to $\mathrm{MH}$.

\section{Notes and references}

a Queensland Micro- and Nanotechnology Center, Griffith University, QLD 4111, Australia

b School of Mechanical and Aerospace Engineering, Nanyang Technological University, Singapore.

* Email: nam-trung.nguyen@griffith.edu.au

1. $\quad$ N. T. Nguyen and S. T. Wereley, Artech House, Boston, 2002.

2. M. Hejazian, W. Lib and N.-T. Nguyen, Lab Chip, 2015, 15, 959. 
3. D. J. Laser and J. G. Santiago, J. Micromech. Microeng., 2004, 14, R35R64.

4. K. A. Au, H. Lai, B. R. Utela and A. Folch, Micromachines, 2011, 2, 179-220.

5. N.-T. Nguyen and Z. Wu, J. Micromech. Microeng., 2005, R1-R16.

6. N. Pamme, Lab Chip, 2007, 7, 644-1659.

7. Y. Chen, P. Li, P.-H. Huang, Y. Xie, J. D. Mai, L. Wang, N.-T. Nguyen and T. J. Huang, Lab Chip, 2014, 14, 626-645.

8. A.G.G. Toh, Z.P. Wang, C.Yang, N.T. Nguyen, Microfluid. Nanofluid., 2014, 16, 1-18.

9. S. Kim, H. J. Kimz and N. L. Jeon, Integr. Biol., 2010, 2, 584-603.

10. W. Siyana, Y. Feng, Z. Lichuan, W. Jiarui, W. Yingyan, J. Li , L. Bingcheng and W. Qi, J. Pharm. Biomed. Anal., 2009, 49, 806-810.

11. J. Diao, L. Young, S. Kim, E. A. Fogarty, S. M. Heilman, P. Zhou, M. L. Shuler, M. Wu and M. P. DeLisa, Lab Chip, 2006, 6, 381-388.

12. Z. Wu, N.-T. Nguyen and X. Huang, J. Micromech. Microeng., 2004 14, 604-611.

13. Z. Wu and N.-T. Nguyen, Sens. Actuators B-Chem., 2005, 107, 965-974.

14. Y. Wang, J. Zhe, B. Chung T. F. and P. Dutta, Microfluid. Nanofluid., 2008, 4, 375-389.

15. T.-H. Tsai, D.-S. Liou, L.-S. Kuo and P.-H. Chen, Sens. Actuator APhys., 2009, 153, 267-273.

16. L. M. Fu, C. H. Tsai, K. P. Leong and C. Y. Wen, 12th International Conference on Magnetic Fluids, Physics Procedia, 2010, 9, 270-273.

17. N. Azimi, M. Rahimi and N. Abdollahi, Chem. Eng. Process., 2015, 97, $12-22$.

18. G.-P. Zhu, M. Hejiazan, X. Huang and N.-T. Nguyen, Lab Chip, 2014, 14, 4609-4615.

19. G.-P. Zhu and N.-T. Nguyen, Microfluid. Nanofluid., 2012, 13, 655-663.

20. M. Hejazian and N.-T. Nguyen, Lab Chip, 2015, 15, 2998.

21. C. Song, N. T. Nguyen, S. H. Tan and A. K. Asundi, Lab Chip, 2009, 9, 1178-1184.

22. D.-T. Phan, C. Yang, N.-T. Nguyen, RSC Adv., 2015, 5, 44336-44341.

23. M. K. Moraveji and M. Hejazian, Numer. Heat Tr. A-APPL, 2014, 66, 315-329.

24. M. K. Moraveji and M. Hejazian, Int. Commun. Heat Mass, 2013, 44, 135-146.

25. C. T. Culbertson, S. C. Jacobson, J. M. Ramsey, Talanta, 2002, 56, 365373.

26. F. R. Ismagilov, A. D. Stroock, P. J. A. Kenis, G. Whitesides and H. A. Stone, Appl. Phys. Lett., 2000, 76, 2376-2378. 